\title{
Modeling and Analysis of Body Potential of Cylindrical Gate-All-Around Nanowire Transistor
}

\author{
Biswajit Ray and Santanu Mahapatra, Member, IEEE
}

\begin{abstract}
A new physically based classical model for the potential distribution of an undoped body cylindrical gate-all-around nanowire transistor is proposed. The model is based on the analytical solution of 2-D Poisson's equation in a cylindrical coordinate system and is valid for both 1 ) weak and strong inversion regimes, 2) long and short-channel transistors, and 3 ) body surfaces and centers. Using the proposed model, for the first time, it is demonstrated that the body potential versus gate voltage characteristics for the devices having equal channel lengths but different body radii pass through a single common point (termed a "crossover point"). It is found that, at this crossover point, there is no potential drop ("pseudo flatband condition") along the radial direction. Using the concept of crossover point, the effect of body radius on the threshold voltage of undoped body multigate transistors is explained. Based on the proposed body potential model, a new compact model for the subthreshold swing is formulated. It is shown that for the devices having very high short-channel effects, the effective subthreshold slope factor is mainly dictated by the potential at the body center rather than that at the surface. All the models are validated against a professional numerical device simulator.
\end{abstract}

Index Terms-Gate all around (GAA), multigate transistor, short-channel effects (SCE), subthreshold slope, undoped body, virtual cathode.

\section{INTRODUCTION}

$\mathbf{T}$ HE UNDOPED cylindrical body gate-all-around (GAA) field effect transistor, which offers maximum gate control over the channel and zero corner effect, is appearing as a promising candidate for sub-45-nm technology nodes [1]-[3]. It is therefore extremely necessary to develop compact models for GAA transistors in order to use them in nanoscale integrated circuit design and simulation.

There is, however, a sharp distinction between the electrostatics of traditional bulk transistors and undoped body devices. In bulk transistors, where the substrate is sufficiently doped, the inversion charges are located close to the surface, and hence, the surface potential solely controls the electrostatic integrity of the device. However, in undoped body devices, the gate electric field penetrates the body center, and inversion charge exists throughout the body. In contrast to the bulk transistors, depending on device geometry, the potential of the body center

Manuscript received March 20, 2008; revised June 3, 2008. This work was supported by the Department of Science and Technology, India, under Grant SR/FTP/ETA-05/2006. The review of this paper was arranged by Editor M. Reed.

The authors are with the Nano Scale Device Research Laboratory, Center for Electronics Design and Technology, Indian Institute of Science, Bangalore 560012, India (e-mail: rbiswajit@cedt.iisc.ernet.in; santanu@cedt.iisc. ernet.in).

Digital Object Identifier 10.1109/TED.2008.927669

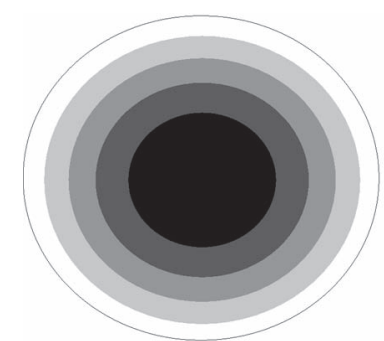

(a)

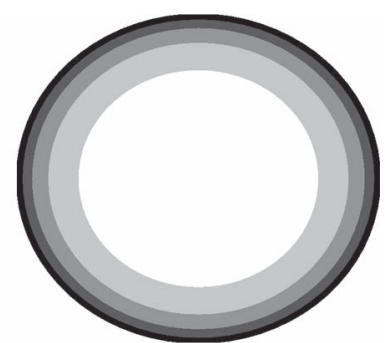

(b)
Fig. 1. Electron concentration at the cross section of GAA nanowire for $R=10 \mathrm{~nm}$ and $L=20 \mathrm{~nm}$. Here, the dark region corresponds to higher mobile charge concentration. (a) $V_{\mathrm{gs}}=0 \mathrm{~V}$. (b) $V_{\mathrm{gs}}=1 \mathrm{~V}$.

of undoped body devices could be higher than the surface in weak inversion regime and the current flows through the center part of the device instead of the surface (see Fig. 1). Several crucial parameters (e.g., subthreshold slope) sometimes become more dependable on the potential of body center rather than that of the surface. Hence, the body-center potential should also be modeled correctly along with the surface potential for an accurate calculation of inversion charge, threshold voltage, and other related parameters of undoped body multigate transistors. Although potential models for GAA transistors [4] and other undoped body multigate transistors [5], [6] have been reported, most of them fail to capture the potential of body center correctly. This is due to the fact that the effect of lateral electric field (short-channel effect) is much more pronounced at the body center than at the surface. Hence, the approximations earlier taken to solve Poisson's equation hold good only at the surface but break down at the body center. Due to the same reasons, most of those models remain valid only in the weak inversion regime.

In this paper, we propose a new physical model for the body potential of GAA transistors which is much more accurate than the previous model [4] and capable of predicting the potential at any point of the body with excellent accuracy in both weak and strong inversion regimes. The model is developed by solving 2-D Poisson's equation in cylindrical coordinate system with some realistic approximations and verified with numerical device simulator (Sentaurus [7]). Using the proposed model, for the first time, we demonstrate that the body potential versus gate voltage characteristics for the devices having equal channel lengths but different body radii pass through a single common point, which is termed as "crossover point." It is found that, at crossover point, there is no potential drop along the radial direction, and hence, the related gate voltage could be treated as "pseudo flatband voltage." Using the concept 


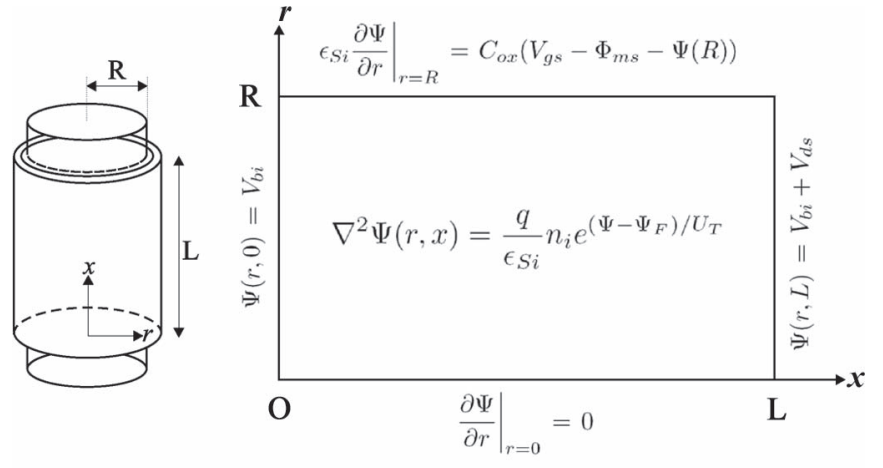

(a)

(b)

Fig. 2. (a) Schematic of cylindrical GAA nanowire transistor. (b) Boundary conditions for Poisson's equation (1) on a cross section starting from body center.

of crossover point, the effect of body radius on the threshold voltage of undoped body multigate transistors is explained. Based on the proposed body potential model, a new model for subthreshold slope is formulated. Some other parameters, e.g., inversion charge, short-channel effect (SCE), and position of virtual cathode, are also studied to demonstrate the impact of body center potential on the electrostatics of GAA transistors.

\section{Model Derivation}

Fig. 2 shows the 3-D structural view of the cylindrical GAA transistor considered in this work. With the assumptions that the channel is undoped and has a large diameter $(>5 \mathrm{~nm})$ to ignore energy quantization, the electrostatics of the channel region is solely governed by the following Poisson's equation, with only mobile charge term included:

$$
\nabla^{2} \Psi(r, x)=\frac{q}{\epsilon_{\mathrm{Si}}} n_{i} e^{\left(\Psi(r, x)-\Psi_{F}\right) / U_{T}} .
$$

The boundary conditions for this partial differential equation (PDE) are shown in Fig. 2, where $\Psi(r, x)$ is the potential at the point $(r, x), q$ is the electronic charge, $n_{i}$ is the intrinsic electron density in silicon, $U_{T}$ is the thermal voltage, $\Phi_{\mathrm{ms}}$ is the gate workfunction referenced to intrinsic silicon, $V_{\mathrm{bi}}$ is the $n^{+}-i$ diode's built-in potential, $C_{\mathrm{ox}}$ is the cylindrical gate oxide capacitance $\left(C_{\mathrm{ox}}=\epsilon_{\mathrm{ox}} /\left(R \ln \left(1+T_{\mathrm{ox}} / R\right)\right)\right.$ [8]), $T_{\mathrm{ox}}$ is the gate oxide thickness, $\epsilon_{\mathrm{ox}}$ and $\epsilon_{\mathrm{Si}}$ are the permittivities of gate oxide and silicon, respectively, $V_{\mathrm{gs}}$ and $V_{\mathrm{ds}}$ are the applied gate and drain voltages, respectively, and $\Psi_{F}$ is the electron quasi-Fermi level. As described in [5] and [6], $\Psi_{F}$ is approximated to be zero throughout the channel for low $V_{\mathrm{ds}}$. It is worth noting that here, we have neglected the hole concentration, and thus, the model is only valid for $\Psi>3 U_{T}$ [10], where hole concentration will be very less compared to electron concentration.

To solve this Poisson's equation, we split the total potential $\Psi$ in the following three components: 1) long-channel potential profile $\Psi_{0}(r) ; 2$ ) Laplace part including all the boundary conditions $\Psi_{L}(r, x)$; and 3$)$ the remaining nonlinear part with all the boundary conditions set to zero $\Psi_{N}(r, x)$

$$
\Psi(r, x)=\Psi_{0}(r)+\Psi_{L}(r, x)+\Psi_{N}(r, x) .
$$

It should be noted that the $\Psi_{N}(r, x)$ component was approximated to be zero in the previous model [4]. In the subsequent sections, we will show that, if the $\Psi_{N}(r, x)$ component is neglected, the model 1) predicts the surface potential quite accurately in weak inversion but overpredicts in the strong and moderate inversion regions, 2) predicts the body center potential quite inaccurately both in weak and strong inversion, and 3 ) does not show any crossover point. However, the subthreshold slope is a weak function of $\Psi_{N}(r, x)$, and the contribution of the $\Psi_{N}(r, x)$ component is only visible for those devices $(2 R / L>1)$ where the SCE is extremely high. The $\Psi_{N}(r, x)$ component is mainly required for the precise calculation of other parameters like inversion charge, current, and threshold voltage where the potential of body center and surface needs to be modeled very accurately.

In order to obtain the total potential profile, we divide the main Poisson's equation (1) in the following three subproblems:

Problem-1

$$
\nabla^{2} \Psi_{0}(r)=\frac{q}{\epsilon_{\mathrm{Si}}} n_{i} e^{\Psi_{0} / U_{T}}
$$

with boundary conditions

$$
\begin{aligned}
\left.\epsilon_{\mathrm{Si}} \frac{\partial \Psi_{0}}{\partial r}\right|_{r=R} & =C_{\mathrm{ox}}\left(V_{\mathrm{gs}}-\Phi_{\mathrm{ms}}-\Psi_{0}(R)\right) \\
\left.\frac{\partial \Psi_{0}}{\partial r}\right|_{r=0} & =0
\end{aligned}
$$

Problem-2

$$
\nabla^{2} \Psi_{L}(r, x)=0
$$

with boundary conditions

$$
\begin{aligned}
\Psi_{L}(r, 0) & =V_{\mathrm{bi}}-\Psi_{0}(r) \\
\Psi_{L}(r, L) & =V_{\mathrm{bi}}+V_{\mathrm{ds}}-\Psi_{0}(r) \\
\left.\epsilon_{\mathrm{Si}} \frac{\partial \Psi_{L}}{\partial r}\right|_{r=R} & =-C_{\mathrm{ox}} \Psi_{L}(R) \\
\left.\frac{\partial \Psi_{L}}{\partial r}\right|_{r=0} & =0
\end{aligned}
$$

Problem-3

$$
\nabla^{2} \Psi_{N}(r, x)=\frac{q}{\epsilon_{\mathrm{Si}}} n_{i} e^{\Psi_{0} / U_{T}}\left(e^{\left(\Psi_{L}+\Psi_{N}\right) / U_{T}}-1\right)
$$

with boundary conditions

$$
\begin{aligned}
\Psi_{N}(r, 0) & =0 \\
\Psi_{N}(r, L) & =0 \\
\left.\epsilon_{\mathrm{Si}} \frac{\partial \Psi_{N}}{\partial r}\right|_{r=R} & =-C_{\mathrm{ox}} \Psi_{N}(R) \\
\left.\frac{\partial \Psi_{N}}{\partial r}\right|_{r=0} & =0 .
\end{aligned}
$$


Problem-1 is an ordinary differential equation (ODE) whose solution is given in

$$
\Psi_{0}(r)=U_{T} \ln \frac{8 B}{\left(1-B r^{2}\right)^{2} \delta}
$$

where $B$ is a parameter whose value depends on $V_{\mathrm{gs}}$ according to (17) which is derived from the boundary condition (4) of Problem-1

$$
V_{\mathrm{gs}}-\Phi_{\mathrm{ms}}=\ln \frac{8 B}{\left(1-B R^{2}\right)^{2} \delta}+\frac{4 U_{T} \epsilon_{\mathrm{Si}} B R}{C_{\mathrm{ox}}\left(1-B R^{2}\right)}
$$

and $\delta$ is a constant whose value is

$$
\delta=\frac{q n_{i}}{\epsilon_{\mathrm{Si}} U_{T}} .
$$

Although the expression of $B$ (17) is implicit, it can be calculated explicitly with high accuracy as shown in [9].

Problem-2 is a mixed boundary value problem which can be solved by variable separation method. As a similar problem is solved in several articles [4], [11], we are giving here the final solution without any derivation. The final solution can be written in terms of an infinite series as follows:

$$
\begin{array}{r}
\Psi_{L}(r, x)=\sum_{n=1}^{\infty}\left[A_{n} \sinh \left(\frac{\mu_{n} x}{R}\right)+B_{n} \sinh \left(\frac{\mu_{n}(L-x)}{R}\right)\right] \\
\times J_{0}\left(\frac{\mu_{n} r}{R}\right)
\end{array}
$$

where $A_{n}$ and $B_{n}$ are given by

$$
\begin{aligned}
A_{n} & =\frac{2 \int_{0}^{R} r\left(V_{\mathrm{bi}}+V_{\mathrm{ds}}-\Psi_{0}(r)\right) J_{0}\left(\frac{\mu_{n} r}{R}\right) d r}{R^{2} \sinh \left(\frac{\mu_{n} L}{R}\right)\left[J_{0}^{2}\left(\mu_{n}\right)+J_{1}^{2}\left(\mu_{n}\right)\right]} \\
& \approx \frac{2\left(V_{\mathrm{bi}}+V_{\mathrm{ds}}-U_{T} \ln \left(\frac{8 B}{\delta}\right)\right) J_{1}\left(\mu_{n}\right)}{\mu_{n} \sinh \left(\frac{\mu_{n} L}{R}\right)\left[J_{0}^{2}\left(\mu_{n}\right)+J_{1}^{2}\left(\mu_{n}\right)\right]} \\
B_{n} & =\frac{2 \int_{0}^{R} r\left(V_{\mathrm{bi}}-\Psi_{0}(r)\right) J_{0}\left(\frac{\mu_{n} r}{R}\right) d r}{R^{2} \sinh \left(\frac{\mu_{n} L}{R}\right)\left[J_{0}^{2}\left(\mu_{n}\right)+J_{1}^{2}\left(\mu_{n}\right)\right]} \\
& \approx \frac{2\left(V_{\mathrm{bi}}-U_{T} \ln \left(\frac{8 B}{\delta}\right)\right) J_{1}\left(\mu_{n}\right)}{\mu_{n} \sinh \left(\frac{\mu_{n} L}{R}\right)\left[J_{0}^{2}\left(\mu_{n}\right)+J_{1}^{2}\left(\mu_{n}\right)\right]} .
\end{aligned}
$$

The eigenvalue $\mu_{n}$ can be found from the following relation which gives infinitely possible values of $\mu$ :

$$
\mu_{n} \frac{J_{1}\left(\mu_{n}\right)}{J_{0}\left(\mu_{n}\right)}=\frac{C_{\mathrm{ox}} R}{\epsilon_{\mathrm{Si}}} .
$$

Here, $J_{0}$ and $J_{1}$ are the Bessel's functions of types zero and one, respectively [12]. Although the expression of $\Psi_{L}(r, x)$ consists of an infinite number of terms, it is explicit w.r.t. $V_{\mathrm{gs}}$, and also, it is found that the higher order $(n>3)$ terms vanish very rapidly.

Problem-3 is a nonlinear second order PDE which is solved in the neighborhood of the boundary $r=0$ (For details of the solving procedure, see the Appendix). The final solution can be given as

$\Psi_{N}(r, x)=\left(\Psi_{L}(r, x)-V_{0}\right)\left(\frac{\cosh \left\{P\left(\frac{L}{2}-x\right)\right\}}{\cosh \left(\frac{P L}{2}\right)}-1\right)$

where $P$ and $V_{0}$ are functions of $r$ as given in

$$
\begin{aligned}
P= & \sqrt{\frac{q n_{i}}{\epsilon_{\mathrm{Si}} U_{T}} e^{\frac{\Psi_{0}(r)}{2 U_{T}}}} \\
V_{0}=U_{T}\left[1+\frac{\Psi_{L}(r, x)+\Psi_{N}\left(r, \frac{L}{2}\right)}{U_{T}}\right. & \left.\quad-\exp \left(\left(\Psi_{L}(r, x)+\Psi_{N}\left(r, \frac{L}{2}\right)\right) /\left(U_{T}\right)\right)\right] .
\end{aligned}
$$

Therefore, the expression of $\Psi_{N}$ is implicit because $V_{0}$ first has to be calculated from (23) at $x=(L / 2)$. However, we solve the resulting equation for $\Psi_{N}(r,(L / 2))$ by Halley's method [14]. By simulation, we observe that the value of $\Psi_{N}(r,(L / 2))$ lies in the range $0--3 U_{T}$, and hence, we take the initial guess for the iteration as $-U_{T}$ which gives excellent accuracy in the first iteration itself. The final expression for $\Psi_{N}(r,(L / 2))$ obtained by Halley's method is given in

$$
\Psi_{N}\left(r, \frac{L}{2}\right) \approx \Psi_{N 0}-\left(1+\frac{1}{2} \frac{L_{f}\left(\Psi_{N 0}\right)}{1-\frac{1}{2} L_{f}\left(\Psi_{N 0}\right)}\right) \frac{f\left(\Psi_{N 0}\right)}{f^{\prime}\left(\Psi_{N 0}\right)}
$$

where

$$
\begin{aligned}
L_{f}\left(\Psi_{N 0}\right) & =\frac{f^{\prime \prime}\left(\Psi_{N 0}\right) f\left(\Psi_{N 0}\right)}{f^{\prime}\left(\Psi_{N 0}\right)^{2}} \\
f\left(\Psi_{N}\right) & =\Psi_{N}-U_{T}\left(1-\cosh \left(\frac{P L}{2}\right)\right)\left(e^{\frac{\Psi_{L}+\Psi_{N}}{U_{T}}}-1\right)
\end{aligned}
$$

where $\Psi_{N 0}\left(=-U_{T}\right)$ is the initial guess. This one step iteration scheme makes the expression of $\Psi_{N}(r, x)$ explicit, and because $\Psi_{0}$ and $\Psi_{L}$ are also explicit, hence, the overall potential $(\Psi(r, x))$ model becomes an explicit function of $V_{\mathrm{gs}}$.

\section{Results And Discussion}

\section{A. Model Verification}

The proposed body potential model is verified against the data obtained from numerical device simulator Sentaurus [7] for different device geometries. From Fig. 3(a)-(d), one can observe that the proposed model predicts the surface as well as the body center potential quite accurately both in the subthreshold and strong inversion regions. It can also be observed [from Fig. 3(e)] that, without the $\Psi_{N}$ component (which is equivalent to the previous model [4]), there is a significant mismatch between simulation- and model-predicted results for body center potential. This mismatch increases with an increasing value of $2 R: L$ ratio, which leads to higher SCEs. Fig. 3(f) shows the 


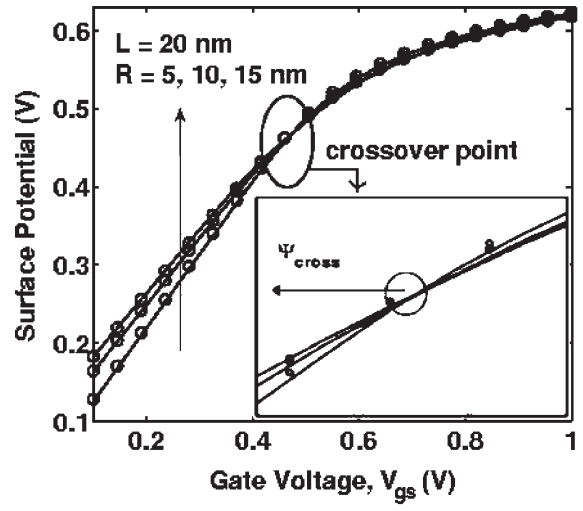

(a)

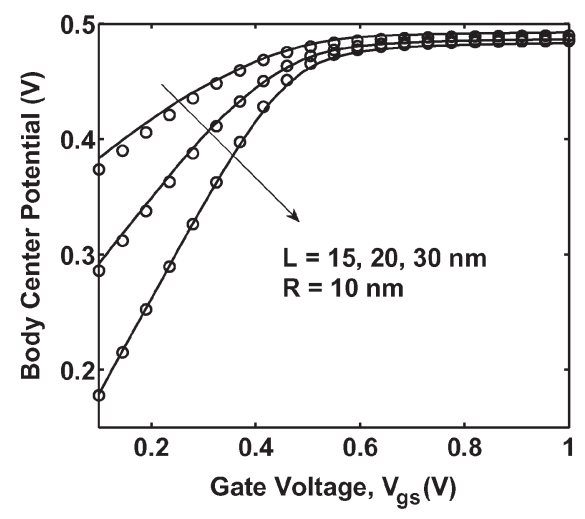

(d)

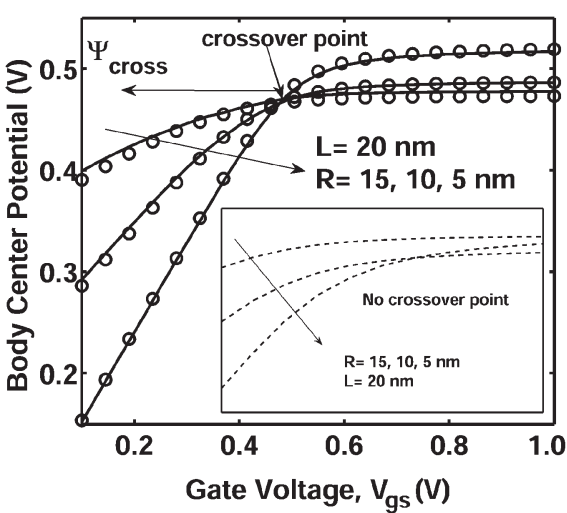

(b)

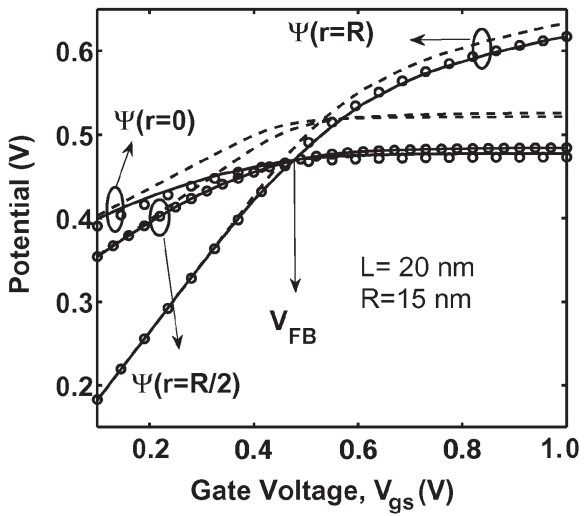

(e)

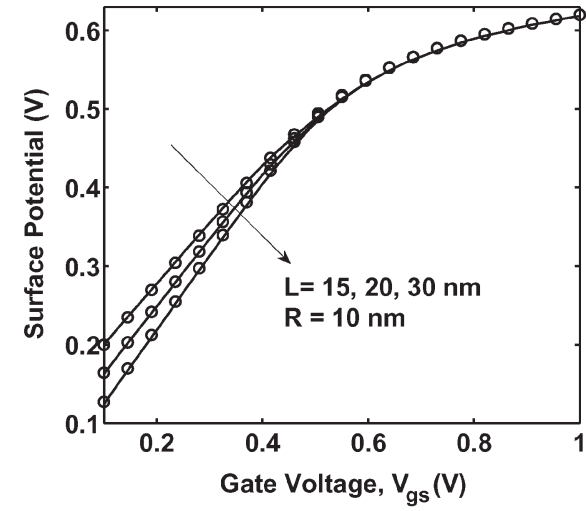

(c)

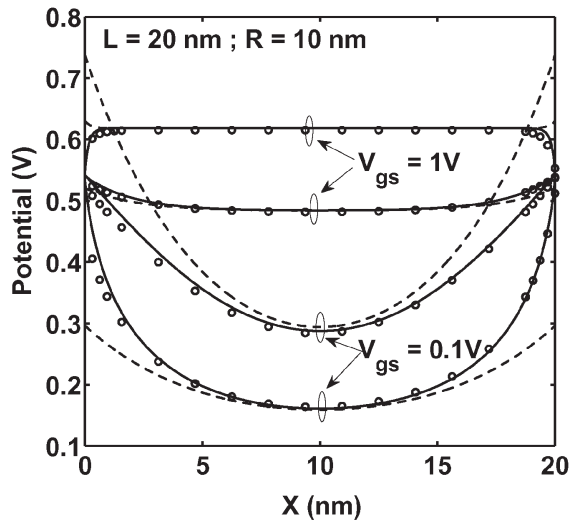

(f)

Fig. 3. Validation of (solid curves) analytical model with the (symbols) numerical simulation results obtained from Sentaurus device simulator. (a) Surface potential $\left(\Psi_{s}\right)$ versus gate voltage $\left(V_{\mathrm{gs}}\right)$ at $x=L / 2$ for a fixed channel length $L=20 \mathrm{~nm}$. (b) Body center potential $\left(\Psi_{b}\right)$ versus gate voltage $\left(V_{\mathrm{gs}}\right)$ at $x=L / 2$ for a fixed channel length $L=20 \mathrm{~nm}$. The same has been shown in the inset without considering the $\Psi_{N}$ part. (c) Surface potential versus gate voltage for a fixed channel radius $R=10 \mathrm{~nm}$. (d) Body center potential versus gate voltage at $x=L / 2$ for a fixed channel radius $R=10 \mathrm{~nm}$. (e) $\Psi_{s}, \Psi_{b}$, and $\Psi(r=R / 2)$ at $x=$ $L / 2$ calculated (solid lines) considering the $\Psi_{N}(r, x)$ term and (dashed lines) not considering the $\Psi_{N}(r, x)$ term. (f) Potential distribution along the channel with only (dashed line) the first term of series solution and (solid lines) ten terms. The top and bottom curves denote the potential distribution at surface and the two intermediate curves are for body center. In all the cases, $V_{\mathrm{ds}}=0 \mathrm{~V}$ and $T_{\mathrm{ox}}=1 \mathrm{~nm}$, midgap gate metal is used.

effect of the number of terms in the series solution on total potential $(\Psi(r, x))$ calculation. We observe that considering only the first term in the series solution introduces considerable error at source and drain boundaries; however, the potential at $L / 2$ (virtual cathode) remains almost unaffected. All the potential versus $V_{\mathrm{gs}}$ characteristics in Fig. 3 are shown considering only the first term of the series solution.

\section{B. Crossover Point and Pseudo Flatband Condition}

The most crucial observation made in this work is the crossover point. From Fig. 3(e), we note that the potential at the surface, body center, and at $r=R / 2$ crosses through a common point for a particular gate voltage $\left(V_{\mathrm{FB}}\right)$. This implies that, at $V_{\mathrm{FB}}$, there is no potential drop along the radial direction from body center to the surface. This is precisely the flatband condition. However, we attribute this situation as pseudo flatband condition because there exists potential variation along the lateral direction. Interestingly, it is also observed that (inset of Fig. 4) $V_{\mathrm{FB}}$ is almost independent of the variation of body radius when channel length is constant, or in other words, devices having the same $L$ but different $R$ hold almost the same value of $V_{\mathrm{FB}}$. Hence, for a given $L$ and different $R$ 's, if we plot potential as a function of $V_{\mathrm{gs}}$ at a particular radial point $(R \cdot m)$,

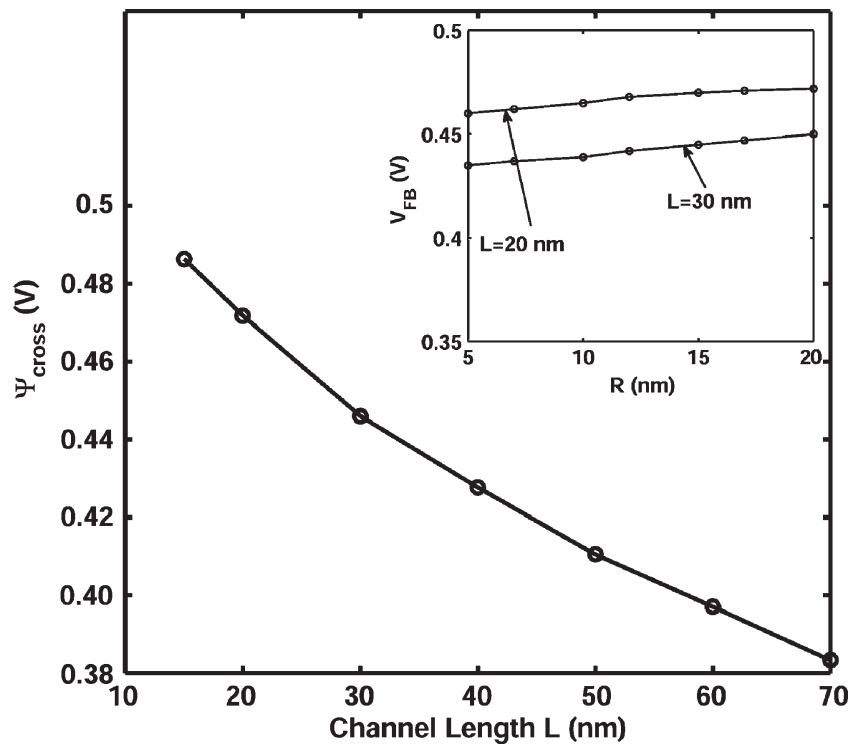

Fig. 4. Change of $\Psi_{\text {cross }}$ as a function of $L$. Inset shows $V_{\mathrm{FB}}$ as a function of $R$.

where $m(<1)$ is a constant, all the characteristics should pass through the common "flatband" point, which we termed as crossover point [Fig. 3(a) and (b)]. The body potential related to 
this point is denoted by $\Psi_{\text {cross. }}$. From Fig. 3(e), we can see that the previous model predicts the $V_{\mathrm{FB}}$ incorrectly, and thus, it also fails to predict the crossover point [inset of Fig. 3(b)]. One more observation made in this work is that the value of $\Psi_{\text {cross }}$ increases with decreasing channel length as shown in Fig. 4. This is due to the fact that, for long-channel devices $\Psi \sim \Psi_{0}$ and for positive $V_{\mathrm{gs}}$ (more precisely for $V_{\mathrm{gs}}>\Phi_{\mathrm{ms}}$ ), $\Psi_{0}(R)>\Psi_{0}(0)$. Hence, for long-channel devices, $V_{\mathrm{FB}} \approx 0$ (or $\Phi_{\mathrm{ms}}$ ). However, for short-channel devices, because of the contribution of $\Psi_{L}$ and $\Psi_{N}, \Psi(R)$ could be less than $\Psi(0)$ for positive $V_{\mathrm{gs}}$. Hence, in order to bring the $\Psi(R)$ equal to $\Psi(0)$, one needs higher gate voltage. As a result, $\Psi_{\text {cross }}$ increases with decreasing $L$. This phenomenon has a critical effect in determining the threshold voltage of the transistor as discussed in the next section.

\section{Effect of Body Radius on Threshold Voltage}

So far, two definitions for threshold voltage $\left(V_{\mathrm{TH}}\right)$ calculation is used for short-channel undoped body transistors. The first one is surface potential based [6], which is similar to the definition of the threshold voltage of bulk devices. The second one [4], [5] is based on the amount of charge per unit area of the body. The charge-based method is argued to be more accurate as, in undoped body transistors, charge exists throughout the body. In the second method, the threshold voltage is defined as the gate voltage at which the charge per unit area $(Q)$ at the virtual cathode becomes equal to some critical threshold charge $\left(Q_{\mathrm{TH}}\right)$, where the $Q$ is defined as

$$
Q=q n_{i} \int_{0}^{R} e^{\Psi\left(r, X_{c}\right) / U_{T}} d r
$$

Here, $X_{c}$ is the position of virtual cathode, which is approximately equal to $L / 2$ for low $V_{\mathrm{ds}}$. Now, as $\Psi\left(r, X_{c}\right)$ is a very complicated function of $r$, the aforementioned integration cannot be evaluated analytically. Therefore, in common practice, the integration is approximated as

$$
Q \approx q n_{i} e^{\Psi\left(R / 2, X_{c}\right) / U_{T}} R
$$

Now, the threshold criteria $Q=Q_{\mathrm{TH}}$ can be expressed in terms of potential as

$$
\Psi\left(R / 2, X_{c}\right)_{\mathrm{TH}}=U_{T} \ln \left(\frac{Q_{\mathrm{TH}}}{q n_{i} R}\right) .
$$

If we use surface potential-based definition (i.e., inversion takes place when $\Psi_{s}$ becomes equal to some constant critical value $\Psi_{\text {crit }}$ ), the relative value between $\Psi_{\text {crit }}$ and $\Psi_{\text {cross }}$ will dictate how the $V_{\mathrm{TH}}$ will change with $R$ for a given channel length. If $\Psi_{\text {crit }}>\Psi_{\text {cross }}$, the threshold voltage will decrease with $R$. However, the opposite trend will be observed for devices having $\Psi_{\text {crit }}<\Psi_{\text {cross }}$. Therefore, surface potential-based definition results in anomalous change in threshold voltage for body radius variation for any given channel length. As $\Psi_{\text {cross }}$ increases with decreasing $L$ (see Fig. 4), it is expected that, for small channel lengths, $V_{\mathrm{TH}}$ should increase with $R$, and for large $L$, it should exhibit the opposite trend. It is worth noting that such anomalous trend was earlier reported by Park and Colinge [1] for doped body devices. However, the similar conclusion cannot
TABLE I

SUBTHRESHOLD SLOPE FACTOR

\begin{tabular}{|c|c|c|c|c|}
\hline $\begin{array}{c}\mathrm{L} \\
(\mathrm{nm})\end{array}$ & $\begin{array}{c}\mathrm{R} \\
(\mathrm{nm})\end{array}$ & $\eta_{I-V}$ & $\eta_{s}$ & $\eta_{b}$ \\
\hline \multirow{3}{*}{20} & 5 & 1.13 & 1.06 & 1.13 \\
\cline { 2 - 5 } & 10 & 1.7 & 1.2 & 1.8 \\
\cline { 2 - 5 } & 15 & 3.1 & 1.22 & 4.43 \\
\hline \multirow{3}{*}{30} & 5 & 1.04 & 1.01 & 1.04 \\
\cline { 2 - 5 } & 10 & 1.2 & 1.1 & 1.2 \\
\cline { 2 - 5 } & 15 & 1.6 & 1.1 & 1.7 \\
\hline \multirow{3}{*}{40} & 5 & 1 & 1 & 1 \\
\cline { 2 - 5 } & 10 & 1.08 & 1 & 1.08 \\
\cline { 2 - 5 } & 15 & 1.2 & 1.05 & 1.3 \\
\hline
\end{tabular}

be drawn for the charge-based definition as the $\Psi\left(R / 2, X_{c}\right)_{\mathrm{TH}}$ varies with $R$.

\section{Modeling of Subthreshold Slope}

The subthreshold slope $(S)$ is defined as

$$
S=2.3 U_{T} \eta\left(r_{\mathrm{eff}}\right)
$$

where $\eta$ is known as subthreshold slope factor. For bulk devices, $\eta$ is calculated from the inverse slope of surface potential versus gate voltage characteristics. However, it is not so straightforward in the case for undoped body devices. In Table I, $\eta_{I V}$ represents the actual subthreshold slope factor extracted from the $I_{d}-V_{\mathrm{gs}}$ plot (obtained from the Sentaurus device simulator), and $\eta_{b}$ and $\eta_{s}$ represent the slope factor value extracted from the $\Psi(0, L / 2)-V_{\mathrm{gs}}$ and $\Psi(R, L / 2)-V_{\mathrm{gs}}$ characteristics, respectively. From this table, it can be seen that for undoped body devices, $\eta$ is a function of radial distance $(r)$, and the effective subthreshold slope factor could be denoted as $\eta\left(r_{\text {eff }}\right)$, where $r_{\text {eff }}$ represents the radial position of the effective conducting path [13].

As observed from Fig. 3(a)-(b), for undoped body devices in subthreshold regime, body center potential could be greater than the surface, and thus, the body center provides the most leaky path for subthreshold current conduction. Hence, if we calculate $\eta$ at body center, it will give the worst case value of $S$. As we can see from Table I, the actual $S$ value will be intermediate of the $S$ value calculated at the surface and at the center. Therefore, in order to accurately model the subthreshold slope, we need to define an effective conducting path somewhere in between surface and center as described in [13]. As a first order approximation, $r_{\text {eff }}=(R / 3)$ is taken in this work, and for this assumption, we get an excellent match with the Sentaurus results as shown in Fig. 5. The expression for $S$ is derived from the potential model as shown in

$$
S=2.3 U_{T} \eta\left(r_{\text {eff }}\right)=\left.2.3 U_{T}\left[\frac{\partial \Psi(r, x)}{\partial V_{g}}\right]^{-1}\right|_{r=r_{\text {eff }}, x=\frac{L}{2}}
$$

where

$$
\frac{\partial \Psi(r, x)}{\partial V_{g}}=\left[1+\alpha+\frac{\alpha\left(1-\cosh \left(\frac{P L}{2}\right)\right)+\left(1-e^{-\frac{\Psi_{L}+\Psi_{N}}{U_{T}}}\right)}{e^{-\frac{\Psi_{L}+\Psi_{N}}{U_{T}}}-\left(1-\cosh \left(\frac{P L}{2}\right)\right)}\right]
$$




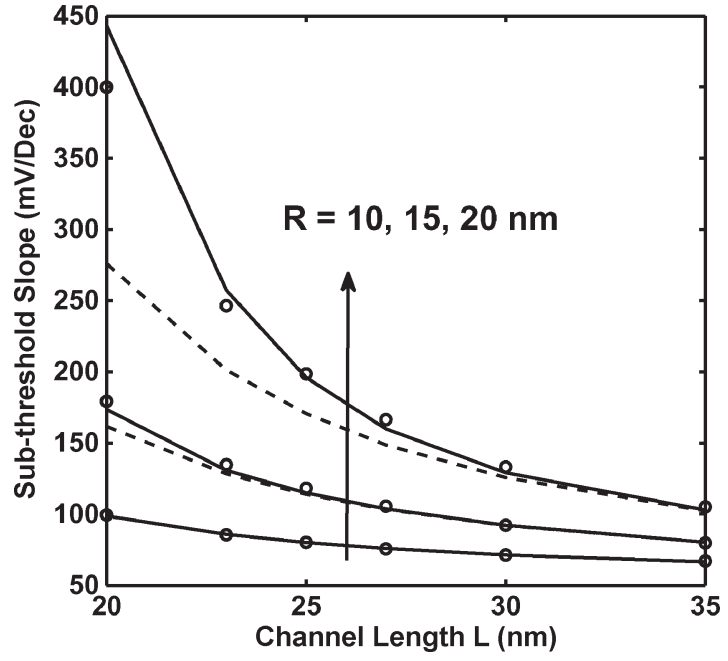

Fig. 5. Subthreshold slope variation with channel length $L$. Symbol represents Sentaurus simulation results and solid line is from model. The dotted line represents the subthreshold slope curve not considering $\Psi_{N}(r, x)$ component.

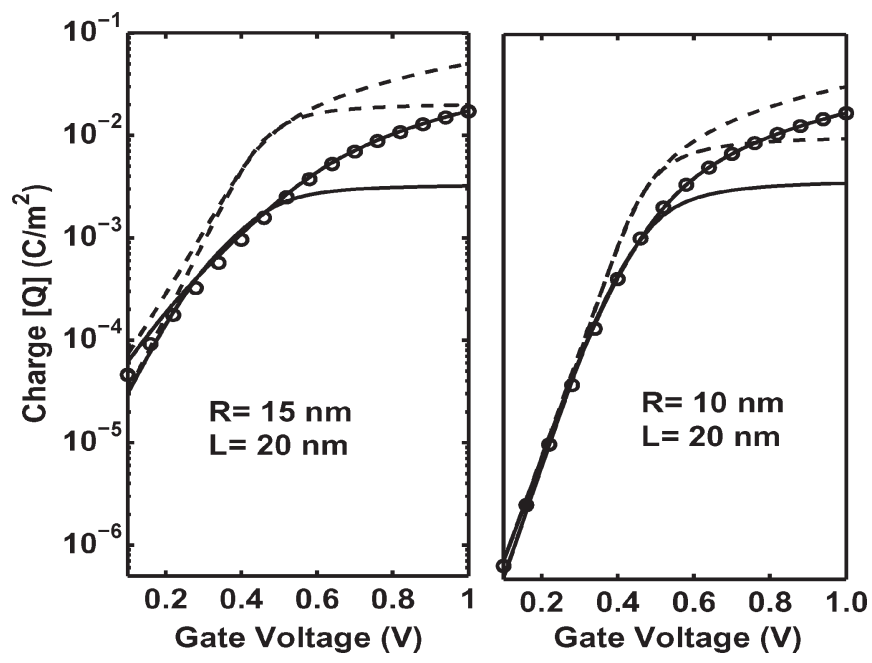

Fig. 6. Inversion charge as a function of gate voltage calculated numerically (29) as well as analytically (30). Symbols represent simulation results, solid lines are for model including $\Psi_{N}$, and the dashed lines are for the model excluding $\Psi_{N}$. Between the two solid lines (as well as the two dashed lines), the lower one represents the analytical calculation of $Q$ (30), whereas the upper one corresponds to the numerical evaluation of $Q$ (29).

with

$$
\alpha=\sum_{n=1}^{\infty}\left[\frac{-4 J_{1}\left(\mu_{n}\right)}{\mu_{n}\left(J_{0}^{2}\left(\mu_{n}\right)+J_{1}^{2}\left(\mu_{n}\right)\right)} \frac{\sinh \left(\frac{\mu_{n} x}{R}\right)}{\sinh \left(\frac{\mu_{n} L}{R}\right)} J_{0}\left(\frac{\mu_{n} r}{R}\right)\right] .
$$

From Fig. 5, it can be seen that the $\Psi_{N}(r, x)$ component plays a significant role in the subthreshold slope expression when SCE is too severe (for device geometry $2 R / L>1$ ).

\section{E. Inversion Charge and SCE}

Inversion charge (coulomb per square meter) at $x=(L / 2)$ is calculated using (29) and (30) with and without considering the $\Psi_{N}$ term in Fig. 6. We observe that the approximation used for the evaluation of the integral (29) is valid in the moderate
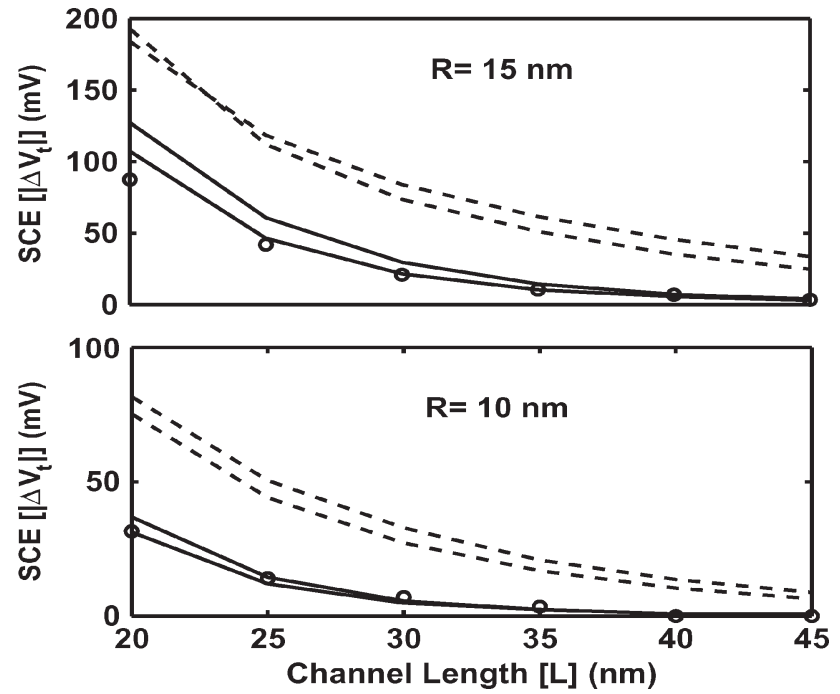

Fig. 7. Magnitude of threshold voltage rolloff due to SCE is plotted for different channel lengths. Symbols represents simulation results, solid lines are for the model including $\Psi_{N}$, and the dashed lines are for the model excluding $\Psi_{N}$. Between the two solid lines (as well as the two dashed lines), the upper one represents the analytical calculation of $Q(30)$, whereas the lower one corresponds to the numerical evaluation of $Q(29)$.

inversion regime, and hence, the analytical expression for $Q$ (30) can be used for the evaluation of $V_{\mathrm{TH}}$ and SCE without incorporating much error, which has been shown in Fig. 7. For the calculation of $V_{\mathrm{TH}}$ and hence SCE, we make use of the charge-based definition as described in Section III-C with $Q_{\mathrm{TH}}=8 \times 10^{-4} \mathrm{C} / \mathrm{m}^{2}$. Both the figures clearly demonstrate the necessity of $\Psi_{N}$ in the calculation of inversion charge and SCE accurately.

\section{F. Virtual Cathode Position}

Virtual cathode point $\left(X_{c}\right)$ denotes the position of minimum potential point in the channel. For low $V_{\mathrm{ds}}$, it is approximately located at $L / 2$, but for higher $V_{\mathrm{ds}}$, it gradually moves toward the source side. Therefore, for high $V_{\mathrm{ds}}$, in order to get an expression of $X_{c}$, we need to solve the equation $(d \Psi / d x)=0$ for a particular $r$. Now, "strictly speaking," our model derivation is not valid for high $V_{\mathrm{ds}}$ as, in that case, the assumption of zero Fermi potential throughout the channel does not hold good. However, for low $V_{\mathrm{gs}}$, neglecting the $\Psi_{N}(r, x)$ term (see the Appendix), it is found that the model can predict the potential profile with acceptable accuracy even for high $V_{\mathrm{ds}}(\sim 1 \mathrm{~V})$. Now, $(d \Psi / d x)=0$ gives us the following:

$$
\begin{aligned}
\sum_{n=1}^{\infty}\left[A_{n} \mu_{n} \cosh \left(\frac{\mu_{n} X_{c}}{R}\right)-B_{n} \mu_{n}\right. & \left.\cosh \left(\frac{\mu_{n}\left(L-X_{c}\right)}{R}\right)\right] \\
& \times J_{0}\left(\frac{\mu_{n} r}{R}\right)=0
\end{aligned}
$$

$X_{c}$ can be evaluated from the aforementioned expression numerically. One interesting observation is made from (36) - that for a given $V_{\mathrm{ds}}$ and $V_{\mathrm{gs}}, X_{c}$ position moves gradually toward the source side as one goes from surface to body center. This is shown in Fig. 8 and validated with the numerical simulation results. We also note from the figure that there is a considerable 


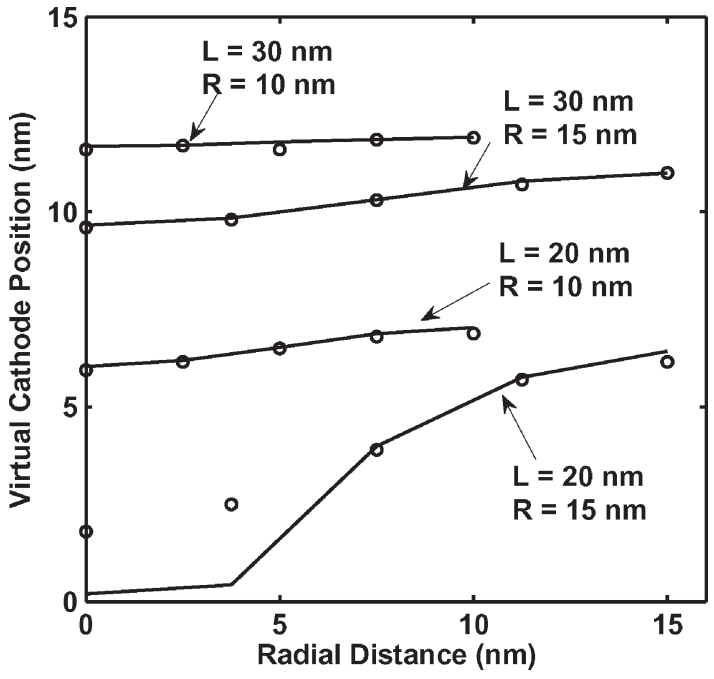

Fig. 8. Virtual cathode position (measured from the source junction) as a function of radial distance for different channel lengths. Symbols represent simulation results and solid lines are for the model. Here, $V_{\mathrm{ds}}=1 \mathrm{~V}$, $V_{\mathrm{gs}}=0.1 \mathrm{~V}, T_{\mathrm{ox}}=1 \mathrm{~nm}$, and midgap gate metal is used.

amount of error between model and simulation for a device with $R=15 \mathrm{~nm}$ and $L=20 \mathrm{~nm}$. This is due to the $\Psi_{N}(r, x)$ term, which cannot be evaluated properly for high $V_{\mathrm{ds}}$ and hence neglected in this derivation. We also observe from the figure that for $(2 R / L)<1, X_{c}$ becomes independent of $r$ and (36) can be simplified to

$$
X_{c}=\frac{R}{2 \mu_{1}}\left(\ln \frac{B_{1}-A_{1} e^{\mu_{1} \mathrm{~L} / \mathrm{R}}}{A_{1} e^{-\mu_{1} \mathrm{~L} / \mathrm{R}}-B_{1}}\right) .
$$

It is worth noting that, if $X_{c}$ varies with $r$, (29)-(31) cannot be used to calculate $V_{\mathrm{TH}}$. Therefore, we need to propose a more careful definition of $V_{\mathrm{TH}}$ in order to correctly evaluate the effect of $V_{\mathrm{ds}}$ variation on it (DIBL). In this scenario, the calculation of $Q(29)$ at $X_{c}(R / 2)$ might give a better result.

\section{CONCLUSION}

In this paper, a new physically based classical model for the potential distribution of undoped body cylindrical GAA nanowire transistors is proposed by solving 2-D Poisson's equation in cylindrical coordinate systems. The proposed model shows excellent accuracy with a professional device simulator in predicting the potential at any point of the body in both weak and strong inversion regimes. Using the proposed model, it is demonstrated that the body potential versus gate voltage characteristics for the devices having equal channel lengths but different body radii pass through a single common crossover point. The concept of crossover point is used to explain the effect of body radius on the threshold voltage of undoped body multigate transistors. A new compact model for the subthreshold slope is formulated from the proposed body potential model. The improvement of the proposed model over the previous model is reflected in subthreshold slope, SCE, and inversion charge calculation.

\section{APPENDIX}

We solve the nonlinear PDE (11) in the neighborhood of the boundary $r=0$ at which both the $r$ dependent terms of the Laplace operator vanish $\left(\left(\partial \Psi_{N} / \partial r\right)=\left(\partial^{2} \Psi_{N} / \partial r^{2}\right)=0\right)$. Hence, the PDE reduces to an ODE of $x$ where the terms dependent on $r$ are treated as constants. Therefore, the resulting ODE takes the following form:

$$
\frac{d^{2} \Psi_{N}}{d x^{2}}=\frac{q}{\epsilon_{\mathrm{Si}}} n_{i} e^{\Psi_{0} / U_{T}}\left(e^{\left(\Psi_{L}+\Psi_{N}\right) / U_{T}}-1\right)
$$

If we treat $\Psi_{L}(r, x)$ also as a constant and substitute $y=$ $\left(\Psi_{L}+\Psi_{N}\right) / U_{T}$, then the aforementioned ODE transforms into following form:

$$
\begin{aligned}
\frac{d^{2} y}{d x^{2}} & =P^{2}\left(e^{y}-1\right) \\
\text { or } \frac{1}{2}\left(\frac{d y}{d x}\right)^{2} & =P^{2}\left(e^{y}-y\right)+C_{0} \\
\text { or } \frac{1}{2}\left(\frac{d y}{d x}\right)^{2} & =y^{\prime \prime}-P^{2} y+P^{2}+C_{0} .
\end{aligned}
$$

Now, we put $y^{\prime}=0$ in the LHS of (40) in order to calculate the value of integration constant $C_{0}$ as $C_{0}=P^{2}\left(y_{\min }-e^{y_{\min }}\right)$. For low $V_{\mathrm{ds}}, y_{\mathrm{min}}$ occurs near $x=L / 2$. However, (41) cannot be solved analytically. Therefore, we approximate (41) by making the LHS zero, and this approximation works very well for the channel region away from S/D boundaries. Hence, the final ODE becomes

$$
y^{\prime \prime}-P^{2} y+P^{2}+C_{0}=0
$$

which can be solved analytically, and the final solution is given in (23).

\section{ACKNOWLEDGMENT}

The authors would like to thank Prof. R. Vittal Rao, Indian Institute of Science, for his valuable suggestions in solving partial differential equations.

\section{REFERENCES}

[1] J. T. Park and J. P. Colinge, "Multiple gate SOI MOSFETs: Device design guidelines," IEEE Trans. Electron Devices, vol. 49, no. 12, pp. 2222-2229, Dec. 2002.

[2] C. P. Auth and J. D. Plummer, "Scaling theory of cylindrical, fullydepleted, surrounding-gate MOSFET's," IEEE Electron Device Lett., vol. 18, no. 2, pp. 74-76, Feb. 1997.

[3] J. Wang, E. Polizzi, and M. Lundstrom, "A computational study of ballistic silicon nanowire transistors," in IEDM Tech. Dig., Dec. 8-10, 2003, pp. 695-698.

[4] H. Abd El Hamid, B. Iniguez, and J. R. Guitart, "Analytical model of the threshold voltage and subthreshold swing of undoped cylindrical gate-allaround-based MOSFETs," IEEE Trans. Electron Devices, vol. 54, no. 3, pp. 572-579, Mar. 2007.

[5] Q. Chen, E. M. Harrell, II, and J. D. Meindl, "A physical short-channel threshold voltage model for undoped symmetric double-gate MOSFETs," IEEE Trans. Electron Devices, vol. 50, no. 7, pp. 1631-1637, Jul. 2003.

[6] Y. Taur, "Analytic solutions of charge and capacitance in symmetric and asymmetric double-gate MOSFETs," IEEE Trans. Electron Devices, vol. 48, no. 12, pp. 2861-2869, Dec. 2001.

[7] Sentaurus Device Version 2006.06, Synopsys Inc.

[8] R. Plonsey and R. E. Collin, Principles and Applications of Electromagnetic Fields. New York: McGraw-Hill, 1961. 
[9] B. Yu, H. Lu, M. Liu, and Y. Taur, "Explicit continuous models for doublegate and surrounding-gate MOSFETs," IEEE Trans. Electron Devices, vol. 54, no. 10, pp. 2715-2722, Oct. 2007.

[10] D. Jimenez, B. Iniguez, J. Sune, L. F. Marsal, J. Pallares, J. Roig, and D. Flores, "Continuous analytic I-V model for surrounding-gate MOSFETs," IEEE Electron Device Lett., vol. 25, no. 8, pp. 571-573, Aug. 2004

[11] Y. Taur and T. H. Ning, Fundamentals of Modern VLSI Devices. Cambridge, U.K.: Cambridge Univ. Press, 1998.

[12] M. Abramowitz and I. Stegun, Handbook of Mathematical Functions. New York: Dover, 1964.

[13] Q. Chen, B. Agrawal, and J. D. Meindl, "A comprehensive analytical subthreshold swing (S) model for double-gate MOSFETs," IEEE Trans. Electron Devices, vol. 49, no. 6, pp. 1086-1090, Jun. 2002.

[14] J. Kou and Y. Li, "Modified Chebyshev-Halley methods with sixthorder convergence," Appl. Math. Comput., vol. 188, no. 1, pp. 681-685, May 2007.

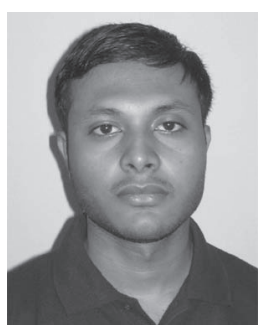

Biswajit Ray received the B.Tech degree in electrical and electronics engineering from National Institute of Technology, Trichy, India, in 2006. He is currently working toward the M.Sc. (engg.) degree in the Nano Scale Device Research Laboratory, Center for Electronics Design and Technology, Indian Institute of Science, Bangalore, India.

His current research interests include compact modeling and simulation of semiconductor devices.

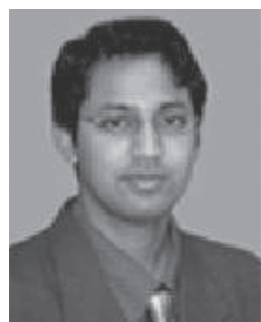

Santanu Mahapatra (M'08) received the B.E. degree in electronics and telecommunication from Jadavpur University, Kolkata, India, in 1999, the M.Tech. degree in electrical engineering with specialization in microelectronics from the Indian Institute of Technology, Kanpur, India, in 2001, and the $\mathrm{Ph} . \mathrm{D}$. degree in electrical engineering from the Swiss Federal Institute of Technology Lausanne, Lausanne, Switzerland, in 2005. In his Ph.D. degree, he worked on the compact modeling of single electron transistors and their hybridization with CMOS.

Since 2005, he has been an Assistant Professor with the Centre for Electronics Design and Technology (CEDT), Indian Institute of Science, Bangalore, India. He is the Founder of the Nano Scale Device Research Laboratory, CEDT, in 2006, where his team is engaged in research on compact modeling and simulation of emerging nanotechnologies and advanced CMOS devices. $\mathrm{He}$ provides consultancy to the device reliability group of Cypress Semiconductor, Bangalore. He is the author and coauthor of several papers published in international journals and refereed conferences. He is also the author of the book Hybrid CMOS Single Electron Transistor Device and Circuit Design (Artech House, 2006). His current research interests include device reliability, multigate transistors, tunnel field-effect transistors, single-electron transistors, and CMOS-nano hybridization.

Dr. Mahapatra is the recipient of the Best Paper Award in the International Semiconductor Conference, Romania, in 2003. He is also the recipient of the IBM Faculty Award and the Microsoft India Research Outstanding Faculty Award in 2007. 